\title{
Ratification vote on taxonomic proposals to the International Committee on Taxonomy of Viruses (2015)
}

\author{
M. J. Adams ${ }^{1}$ E. J. Lefkowitz ${ }^{2}$ A. M. Q. King ${ }^{3}$ - D. H. Bamford ${ }^{4}$. \\ M. Breitbart ${ }^{5}$ - A. J. Davison ${ }^{6}$ S. A. Ghabrial ${ }^{7}$ - A. E. Gorbalenya ${ }^{8}$. \\ N. J. Knowles ${ }^{9}$ P. Krell ${ }^{10}$ - R. Lavigne ${ }^{11}$ - D. Prangishvili ${ }^{12} \cdot$ H. Sanfaçon ${ }^{13}$ \\ S. G. Siddell ${ }^{14}$ P. Simmonds ${ }^{15} \cdot$ E. B. Carstens ${ }^{16}$
}

Published online: 28 April 2015

(C) Springer-Verlag Wien 2015

\begin{abstract}
Changes to virus taxonomy approved and ratified by the International Committee on Taxonomy of Viruses in February 2015 are listed.
\end{abstract}

Changes to virus taxonomy (the Universal Scheme of Virus Classification of the International Committee on Taxonomy of Viruses [ICTV]) now take place annually and are the result of a multi-stage process. In accordance with the ICTV Statutes, proposals submitted to the Executive Committee undergo a review process that involves input from the ICTV Study Groups (SGs) and Subcommittees (SCs), other interested virologists, and the ICTV Executive Committee. Reviewed proposals are then presented for

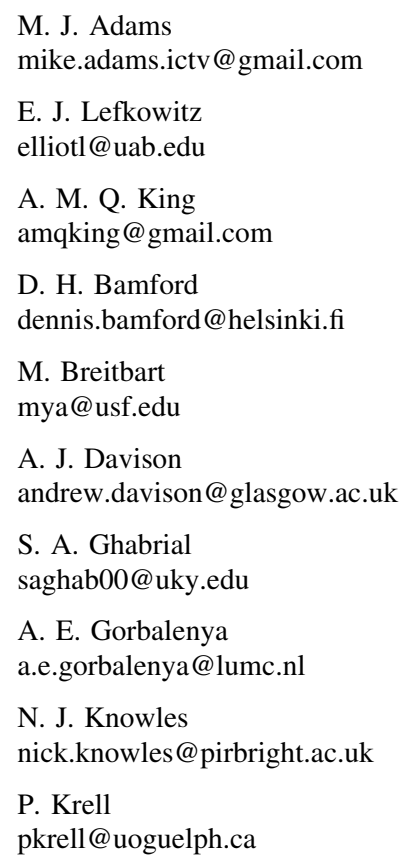

ratification to the full ICTV membership by publication on an ICTV web site followed by an electronic vote. The latest list of approved proposals was prepared and presented on the ICTV online web pages by November 2014. This list of proposals was then sent via email on 20 January 2015 to the 146 members of ICTV, namely the ICTV Executive Committee Members, Life Members, ICTV Subcommittee Members and ICTV National Representatives. Members were then requested to vote on whether or not to ratify the taxonomic proposals (voting closed on 20 February 2015).

This report lists the taxonomic proposals that were ratified by ICTV members. Note that by ICTV convention, all approved taxon names are italicized. The

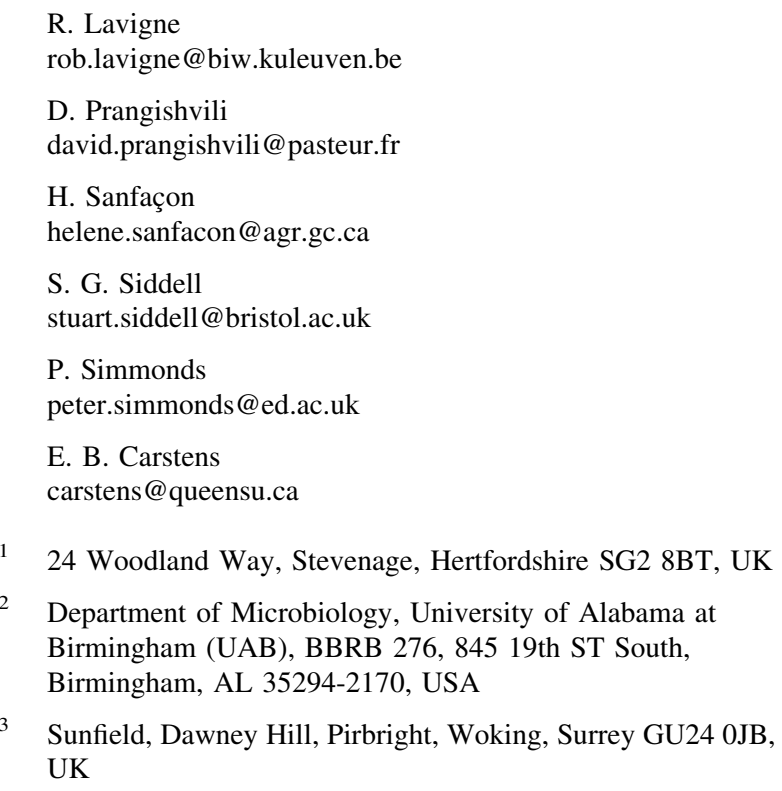


proposals are listed by Subcommittee, and then the filename as posted on-line. For the first time, each proposal is formally cited so that a list of the authors appears in the
References. This provides a means of acknowledging their efforts and also links to the specific proposal on the ICTV web site.

\section{Invertebrate virus subcommittee}

\subsection{2aI.A.v3.Betaentomopoxvirus_3sp [1]}

2013.002aI create 3 species, Adoxophyes honmai entomopoxvirus, Choristoneura rosaceana entomopoxvirus and Mythimna separata entomopoxvirus in the genus Betaentomopoxvirus, subfamily Entomopoxvirinae, family Poxviridae

\subsection{1aI.A.v1. Entomopoxvirus-spcorr [2]}

2014.001aI Correct typographical and other errors in species names in the subfamily Entomopoxvirinae:

\section{Old name}

(a) in genus Alphaentomopoxvirus:

Figulus subleavis entomopoxvirus

Demodema boranensis entomopoxvirus

(b) in genus Betaentomopoxvirus:

Acrobasis zelleri entomopoxvirus ' $L$ '

Amsacta moorei entomopoxvirus ' $L$ '

Arphia conspersa entomopoxvirus ' $O$ '

Choristoneura biennis entomopoxvirus ' $L$ '

Choristoneura conflicta entomopoxvirus ' $L$ '

Choristoneura diversuma entomopoxvirus ' $L$ '

Choristoneura fumiferana entomopoxvirus ' $L$ '

Chorizagrotis auxiliars entomopoxvirus ' $L$ '

Heliothis armigera entomopoxvirus ' $L$ '

Locusta migratoria entomopoxvirus ' $O$ '

Oedaleus senigalensis entomopoxvirus ' $O$ '

Operophtera brumata entomopoxvirus ' $L$ '

Schistocera gregaria entomopoxvirus ' $O$ '

(c) in genus Gammaentomopoxvirus:

Goeldichironomus haloprasimus entomopoxvirus
New name

Figulus sublaevis entomopoxvirus

Demodema bonariensis entomopoxvirus

Acrobasis zelleri entomopoxvirus

Amsacta moorei entomopoxvirus

Arphia conspersa entomopoxvirus

Choristoneura biennis entomopoxvirus

Choristoneura conflicta entomopoxvirus

Choristoneura diversuma entomopoxvirus

Choristoneura fumiferana entomopoxvirus

Chorizagrotis auxiliaris entomopoxvirus

Heliothis armigera entomopoxvirus

Locusta migratoria entomopoxvirus

Oedaleus senegalensis entomopoxvirus

Operophtera brumata entomopoxvirus

Schistocerca gregaria entomopoxvirus

Goeldichironomus holoprasinus entomopoxvirus
4 Institute of Biotechnology and Department of Biosciences, University of Helsinki, Viikinkaari 5, 00014 Helsinki, Finland

5 College of Marine Science, University of South Florida, 140 7th Avenue South, Saint Petersburg, FL 33701, USA

6 MRC-University of Glasgow Centre for Virus Research, Sir Michael Stoker Building, 464 Bearsden Road, Glasgow G61 1QH, UK

7 Department of Plant Pathology, University of Kentucky, 201F Plant Science Building, 1405 Veterans Drive, Lexington, KY 40546-0312, USA

8 Department of Medical Microbiology, Leiden University Medical Center, E4-P, rm. E4-72, P.O. Box 9600, 2300 RC Leiden, The Netherlands

9 The Pirbright Institute, Ash Road, Pirbright, Surrey GU24 0NF, UK

10 Department of Molecular and Cellular Biology, University of Guelph, 50 Stone Road East, Guelph, ON N1G 2W1, Canada
11 Laboratory of Gene Technology, Katholieke Universiteit Leuven, Kasteelpark Arenberg 21, Box 2462, 3001 Louvain, Belgium

12 Biologie Moléculaire du Gène chez les Extrêmophiles, Institut Pasteur, 25, rue du Dr. Roux, 75724 Paris Cedex 15, France

13 Agriculture and Agri-Food Canada, Pacific Agri-Food Research Centre, 4200 Highway 97, Summerland, BC V0H 1Z0, Canada

14 Department of Cellular and Molecular Medicine, Medical and Veterinary Sciences, University of Bristol, University Walk, Bristol BS8 1TD, UK

15 Infection and Immunity Division, Roslin Institute, University of Edinburgh, Easter Bush, Edinburgh EH25 9RG, UK

16 Department of Biomedical and Molecular Sciences, Queen's University, Kingston, ON K7L 3N6, Canada 
2014.002aI.A.v1. Alphabaculovirus-sprem [3]

2014.002aI remove species Helicoverpa zea single nucleopolyhedrovirus from the genus Alphabaculovirus

Plant virus subcommittee

2013.010a,bP.A.v2.Umbravirus_move [4]

2013.010aP remove genus Umbravirus from its present (unassigned) position

2013.010bP reassign genus Umbravirus to the family Tombusviridae

2014.001aP.A.v1.Tobamovirus_2sp [5]

2014.001aP create 2 species, Tomato mottle mosaic virus and Yellow tailflower mild mottle virus, in the genus Tobamovirus, family Virgaviridae

2014.002aP.A.v1.Pospiviroid_sprem [6]

2014.002aP remove (abolish) the species Mexican papita viroid (currently in the genus Pospiviroid, family Pospiviroidae)

2014.003aP.A.v2.Hostuviroid_sp [7]

2014.003aP create species Dahlia latent viroid in the genus Hostuviroid

2014.004aP.A.v2.Torradovirus_sp [8]

2014.004aP create species Lettuce necrotic leaf curl virus in the genus Torradovirus, family Secoviridae, order Picornavirales

2014.005aP.A.v1.Tritimovirus_sp [9]

2014.005aP create the species Tall oatgrass mosaic virus in the genus Tritimovirus, family Potyviridae

2014.006aP.A.v2.Tombusviridae_4sp [10]

$2014.006 \mathrm{aP} \quad$ create 4 species (Elderberry latent virus, Pelargonium chlorotic ring pattern virus, Pelargonium ringspot virus and Rosa rugosa leaf distortion virus) to be unassigned in the family Tombusviridae

2014.007aP.A.v1.Panicovirus_sp [11]

2014.007aP create species Thin paspalum asymptomatic virus in genus Panicovirus in the family Tombusviridae

2014.008aP.A.v3.Tombusviridae_sp [12]

2014.006aP create species Trailing lespedeza virus 1 to be unassigned in the family Tombusviridae

2014.009aP.A.v4.Potyvirus_12sp [13]

2014.009aP create 12 species (Bidens mosaic virus, Blue squill virus A, Brugmansia mosaic virus, Calla lily latent virus, Habenaria mosaic virus, Keunjorong mosaic virus, Lupinus mosaic virus, Panax virus $Y$, Tomato necrotic stunt virus, Cyrtanthus elatus virus A, Verbena virus $Y$ and Zucchini tigre mosaic virus) in the genus Potyvirus, family Potyviridae

2014.010a-dP.A.v2.Rosadnavirus [14]

2014.010aP create species Rose yellow vein virus in genus Rosadnavirus (new) in the family Caulimoviridae

$2014.010 \mathrm{bP} \quad$ create a new genus

$2014.010 \mathrm{cP} \quad$ name the genus Rosadnavirus

2014.010dP designate Rose yellow vein virus as type species of the new genus

2014.011aP.A.v1.Caulimovirus_sp [15]

2014.011aP create species Soybean Putnam virus in genus Caulimovirus, family Caulimoviridae

2014.012aP.A.v1.Badnavirus_7sp [16]

2014.012aP create 7 species (Banana streak IM virus, Banana streak UA virus, Banana streak UI virus, Banana streak UL virus, Banana streak UM virus, Fig badnavirus 1 and Pagoda yellow mosaic associated virus) in the genus Badnavirus, family Caulimoviridae

2014.013aP.A.v2.Emaravirus_sp [17]

2014.013aP create species Pigeonpea sterility mosaic virus in the genus Emaravirus

Prokaryote virus subcommittee

2012.002a-dB.A.v3.Corndoglikevirus [18]

2012.002aB create 2 species (Mycobacterium phage corndog and Mycobacterium phage firecracker) in the genus Corndoglikevirus (new), family Siphoviridae, order Caudovirales

2012.002bB create a new genus in the family Siphoviridae

2012.002cB name the new genus Corndoglikevirus

$2012.002 \mathrm{~dB}$ designate Mycobacterium phage corndog as the type species of the new genus

2012.003a-dB.A.v3.D3112likevirus [19]

$2012.003 \mathrm{aB}$ 
create 8 species (Pseudomonas phage D3112, Pseudomonas phage Dms3, Pseudomonas phage Fha0480, Pseudomonas phage Lpb1, Pseudomonas phage Mp22, Pseudomonas phage Mp29, Pseudomonas phage Mp38 and Pseudomonas phage Palphi) in the genus D3112likevirus (new), family Siphoviridae, order Caudovirales

2012.003bB create a new genus in the family Siphoviridae

2012.003cB name the new genus D3112likevirus

$2012.003 \mathrm{~dB}$ designate Pseudomonas phage D3112 as the type species of the new genus

2013.001a-oB.A.v5.Sphaerolipoviridae [20, 21]

2013.001aB create 3 species (Haloarcula hispanica virus SH1, Haloarcula hispanica virus PH1 and Haloarcula hispanica icosahedral virus 2) in the genus Alphasphaerolipovirus (new), family Sphaerolipoviridae (new)

$2013.001 \mathrm{bB}$ create a new genus in the family Sphaerolipoviridae (new)

2013.001cB name the new genus Alphasphaerolipovirus

$2013.001 \mathrm{~dB}$ designate Haloarcula hispanica virus SH1 as the type species of the newus

2013.001 B B create species Natrinema virus SNJ1 in the genus Betasphaerolipovirus (new), family Sphaerolipoviridae (new)

2013.001fB create a new genus in the family Sphaerolipoviridae (new)

2013.001gB name the new genus Betasphaerolipovirus

$2013.001 \mathrm{hB}$ designate Natrinema virus $S N J 1$ as the type species of the new genus

2013.001iB create 2 species, Thermus thermophilus phage P23-77 and Thermus thermophilus phage IN93, in the genus

Gammasphaerolipovirus (new), family Sphaerolipoviridae (new)

2013.001jB create a new genus in the family Sphaerolipoviridae (new)

$2013.001 \mathrm{kB}$ name the new genus Gammasphaerolipovirus

2013.0011B designate Thermus thermophilus phage P23-77 as the type species of the new genus

$2013.001 \mathrm{mB}$ create a new unassigned family

$2013.001 \mathrm{nB}$ name the new family Sphaerolipoviridae

2013.001oB assign 3 genera, Alphasphaerolipovirus, Betasphaerolipovirus and Gammasphaerolipovirus, to the new family

2013.004a-kB.A.v4.Eucampyvirinae [22, 23]

$2013.004 \mathrm{aB}$ create 4 species (Campylobacter phage CP220, Campylobacter phage CPt10, Campylobacter phage IBB35 and Campylobacter phage CP21) in the genus Cp220likevirus (new), subfamily Eucampyvirinae (new), family Myoviridae, order Caudovirales

2013.004bB create 3 species (Campylobacter phage CP81, Campylobacter phage CPX and Campylobacter phage NCTC12673) in the genus Cp8unalikevirus (new), subfamily Eucampyvirinae (new), family Myoviridae, order Caudovirales

2013.004cB create a new genus in the subfamily Eucampyvirinae (new), family Myoviridae

$2013.004 \mathrm{~dB}$ name the new genus Cp220likevirus

$2013.004 \mathrm{eB}$ designate Campylobacter phage CP220 as the type species of the new

2013.004fB create a new genus in the subfamily Eucampyvirinae (new), family Myoviridae

2013.004gB name the new genus Cp8unalikevirus

2013.004hB designate Campylobacter phage CP81 as the type species of the new genus

2013.004iB create a new subfamily in the family Myoviridae

2013.004jB name the new subfamily Eucampyvirinae

$2013.004 \mathrm{kB}$ assign 2 genera, Cp220likevirus and Cp8unalikevirus, to the new subfamily

2013.005a-dB.A.v3.Phiflikevirus [24]

2013.005aB create 3 species (Enterococcus phage phifl, Enterococcus phage phifl 2 and Enterococcus phage phifl3) in the genus Phiflikevirus (new), family Siphoviridae, order Caudovirales

2013.005bB create a new genus in the family Siphoviridae

$2013.005 \mathrm{cB}$ name the new genus Phiflikevirus

$2013.005 \mathrm{~dB}$ designate Enterococcus phage phifl as the type species of the new genus

2013.006a-dB.A.v3.Iebhlikevirus [25]

2013.006aB create 2 species, Bacillus phage IEBH and Bacillus phage 250, in the genus Iebhlikevirus (new), family Siphoviridae, order Caudovirales

2013.006bB create a new genus in the family Siphoviridae

$2013.006 \mathrm{cB}$ name the new genus Iebhlikevirus

$2013.006 \mathrm{~dB}$ designate Bacillus phage IEBH as the type species of the new gen

2013.007a-dB.A.v3.Halolikevirus [26]

2013.007aB create 2 species, Mycobacterium phage halo and Mycobacterium phage liefie, in the genus Halolikevirus (new), family Siphoviridae, order Caudovirales 
2013.007bB create a new genus in the family Siphoviridae

$2013.007 \mathrm{cB}$ name the new genus Halolikevirus

$2013.007 \mathrm{~dB}$ designate Mycobacterium phage halo as the type species of the new genus

\subsection{8a-dB.A.v3.Che8likevirus [27]}

2013.008aB create the following 28 species in the genus Che8likevirus (new), family Siphoviridae, order Caudovirales: Mycobacterium phage Che8, Mycobacterium phage PMC, Mycobacterium phage mutaforma13, Mycobacterium phage dotproduct, Mycobacterium phage gumbie, Mycobacterium phage Llij, Mycobacterium phage spartacus, Mycobacterium phage Pacc40, Mycobacterium phage ibhubesi, Mycobacterium phage tweety, Mycobacterium phage taj, Mycobacterium phage wee, Mycobacterium phage dorothy, Mycobacterium phage deadp, Mycobacterium phage SG4, Mycobacterium phage boomer, Mycobacterium phage dlane, Mycobacterium phage ardmore, Mycobacterium phage fruitloop, Mycobacterium phage shilan, Mycobacterium phage drago, Mycobacterium phage ramsey, Mycobacterium phage rockyhorror, Mycobacterium phage mozy, Mycobacterium phage shauna1, Mycobacterium phage Che9d, Mycobacterium phage avani and Mycobacterium phage yoshi

2013.008bB create a new genus in the family Siphoviridae

$2013.008 \mathrm{cB}$ name the new genus Che8likevirus

$2013.008 \mathrm{~dB}$ designate Mycobacterium phage Che 8 as the type species of the new genus

\subsection{9a-dB.A.v3.Bignuzlikevirus [28]}

2013.009aB create 2 species, Mycobacterium phage bignuz and Mycobacterium phage jebeks, in the genus Bignuzlikevirus (new), family Siphoviridae, order Caudovirales

2013.009bB create a new genus in the family Siphoviridae

2013.009cB name the new genus Bignuzlikevirus

$2013.009 \mathrm{~dB}$ designate Mycobacterium phage bignuz as the type species of the new genus

\subsection{0a-dB.A.v3.Phijlunalikevirus [29]}

2013.010aB create 3 species (Lactobacillus phage phijl1, Pediococcus phage clp1 and Lactobacillus phage ATCC8014) in the genus Phijlunalikevirus (new), family Siphoviridae, order Caudovirales

2013.010bB create a new genus in the family Siphoviridae

2013.010cB name the new genus Phijlunalikevirus

2013.010dB designate Lactobacillus phage phijl1 as the type species of the new genus

\subsection{1a-dB.A.v3.Barnyardlikevirus [30]}

$2013.011 \mathrm{aB}$ create 4 species (Mycobacterium phage barnyard, Mycobacterium phage predator, Mycobacterium phage konstantine and Mycobacterium phage patience) in the genus Barnyardlikevirus (new), family Siphoviridae, order Caudovirales

$2013.011 \mathrm{bB}$ create a new genus in the family Siphoviridae

2013.011cB name the new genus Barnyardlikevirus

$2013.011 \mathrm{~dB}$ designate Mycobacterium phage barnyard as the type species of the new

\subsection{2a-dB.A.v3.Charlielikevirus [31]}

2013.012aB create 2 species, Mycobacterium phage charlie and Mycobacterium phage redi, in the genus Charlielikevirus (new), family Siphoviridae, order Caudovirales

2013.012bB create a new genus in the family Siphoviridae

2013.012cB name the new genus Charlielikevirus

2013.012dB designate Mycobacterium phage charlie as the type species of the newus

\subsection{3a-dB.A.v3.Che9clikevirus [32]}

$2013.013 \mathrm{aB} \quad$ create 3 species (Mycobacterium phage Che 9 c, Mycobacterium phage brujita and Mycobacterium phage babsiella) in the genus Che9clikevirus (new), family Siphoviridae, order Caudovirales

2013.013bB create a new genus in the family Siphoviridae

2013.013cB name the new genus Che9clikevirus

2013.013dB designate Mycobacterium phage Che $9 c$ as the type species of the newus

2013.014a-dB.A.v3.Cjwunalikevirus [33]

2013.014aB create 9 species (Mycobacterium phage CJW1, Mycobacterium phage pumpkin, Mycobacterium phage 244, Mycobacterium phage porky, Mycobacterium phage Bask21, Mycobacterium phage sirduracell, Mycobacterium phage eureka, Mycobacterium phage kostya and Mycobacterium phage toto) in the genus Cjwunalikevirus (new), family Siphoviridae, order Caudovirales

2013.014bB create a new genus in the family Siphoviridae

2013.014cB name the new genus Cjwunalikevirus

$2013.014 \mathrm{~dB}$ designate Mycobacterium phage CJW1 as the type species of the new genus

2013.015a-dB.A.v3.D3likevirus [34]

2013.015aB create 2 species, Pseudomonas phage D3 and Pseudomonas phage PMG1, in the genus D3likevirus (new), family Siphoviridae, order Caudovirales 
2013.015bB create a new genus in the family Siphoviridae

$2013.015 \mathrm{cB}$ name the new genus D3likevirus

2013.015dB designate Pseudomonas phage D3 as the type species of the new genus

\subsection{6aB.A.v3.L5likevirus_62sp [35]}

2013.016aB create the following 62 species in the genus L5likevirus, family Siphoviridae, order Caudovirales: Mycobacterium phage L5, Mycobacterium phage SWU1, Mycobacterium phage Che12, Mycobacterium phage D29, Mycobacterium phage trixie, Mycobacterium phage turbido, Mycobacterium phage Ta17a, Mycobacterium phage redrock, Mycobacterium phage pukovnik, Mycobacterium phage ericb, Mycobacterium phage hammer, Mycobacterium phage gladiator, Mycobacterium phage jeffabunny, Mycobacterium phage Bxb1, Mycobacterium phage billknuckles, Mycobacterium phage switzer, Mycobacterium phage kssjeb, Mycobacterium phage U2, Mycobacterium phage mrgordo, Mycobacterium phage museum, Mycobacterium phage lesedi, Mycobacterium phage solon, Mycobacterium phage bethlehem, Mycobacterium phage KBG, Mycobacterium phage kugel, Mycobacterium phage bruns, Mycobacterium phage violet, Mycobacterium phage euphoria, Mycobacterium phage BPBiebs31, Mycobacterium phage nepal, Mycobacterium phage doom, Mycobacterium phage marcell, Mycobacterium phage lockley, Mycobacterium phage skipole, Mycobacterium phage JC27, Mycobacterium phage Jasper, Mycobacterium phage Ridgecb, Mycobacterium phage Perseus, Mycobacterium phage Bxz2, Mycobacterium phage JHC117, Mycobacterium phage microwolf, Mycobacterium phage wonder, Mycobacterium phage heldan, Mycobacterium phage rockstar, Mycobacterium phage peaches, Mycobacterium phage arturo, Mycobacterium phage LHTSCC, Mycobacterium phage backyardigan, Mycobacterium phage benedict, Mycobacterium phage george, Mycobacterium phage cuco, Mycobacterium phage tiger, Mycobacterium phage timshel, Mycobacterium phage saintus, Mycobacterium phage astro, Mycobacterium phage packman, Mycobacterium phage alma, Mycobacterium phage rebeuca, Mycobacterium phage goose, Mycobacterium phage twister, Rhodococcus phage RGL3 and Rhodococcus phage RER2

2013.017a-dB.A.v3.Bronlikevirus [36]

2013.017aB create 4 species (Mycobacterium phage bron, Mycobacterium phage joedirt, Mycobacterium phage rumpelstiltskin and Mycobacterium phage Faith1) in the genus Bronlikevirus (new), family Siphoviridae, order Caudovirales

2013.017bB create a new genus in the family Siphoviridae

$2013.017 \mathrm{cB}$ name the new genus Bronlikevirus

2013.017dB designate Mycobacterium phage bron as the type species of the new genus

2013.018a-dB.A.v3.Omegalikevirus [37]

$2013.018 \mathrm{aB} \quad$ create 6 species (Mycobacterium phage omega, Mycobacterium phage littlee, Mycobacterium phage courthouse, Mycobacterium phage optimus, Mycobacterium phage baka and Mycobacterium phage thibault) in the genus Omegalikevirus (new), family Siphoviridae, order Caudovirales

2013.018bB create a new genus in the family Siphoviridae

$2013.018 \mathrm{cB}$ name the new genus Omegalikevirus

2013.018dB designate Mycobacterium phage omega as the type species of the new genus

\subsection{9a-dBA.v4.Pbiunalikevirus [38]}

2013.019aB create species Mycobacterium phage PBI1 in the genus Pbiunalikevirus (new), family Siphoviridae, order Caudovirales

2013.019bB create a new genus in the family Siphoviridae

2013.019cB name the new genus Pbiunalikevirus

2013.019dB designate Mycobacterium phage PBI1 as the type species of the new genus

2013.020a-dB.A.v4.Pgonelikevirus [39]

$2013.020 \mathrm{aB} \quad$ create 12 species (Mycobacterium phage Pg1, Mycobacterium phage oline, Mycobacterium phage rosebush, Mycobacterium phage pipefish, Mycobacterium phage gadjet, Mycobacterium phage athena, Mycobacterium phage cooper, Mycobacterium phage chrisnmich, Mycobacterium phage stinger, Mycobacterium phage nigel, Mycobacterium phage zemanar and Mycobacterium phage acadian) in the genus Pgonelikevirus (new), family Siphoviridae, order Caudovirales

2013.020bB create a new genus in the family Siphoviridae

$2013.020 \mathrm{cB}$ name the new genus Pgonelikevirus

2013.020dB designate Mycobacteriophage Pgl as the type species of the new genus

2013.021a-dB.A.v3.Reylikevirus [40]

2013.021aB create 2 species, Mycobacterium phage rey and Mycobacterium phage bongo, in the genus Reylikevirus (new), family Siphoviridae, order Caudovirales

2013.021bB create a new genus in the family Siphoviridae

$2013.021 \mathrm{cB} \quad$ name the new genus Reylikevirus

$2013.021 \mathrm{~dB} \quad$ designate Mycobacterium phage rey as the type species of the new genus

2013.022a-dB.A.v3.Tm4likevirus [41]

2013.022aB create 9 species (Mycobacterium phage TM4, Mycobacterium phage angelica, Mycobacterium phage jaws, Mycobacterium phage crimd, Mycobacterium phage anaya, Mycobacterium phage pixie, Mycobacterium phage macncheese, Mycobacterium phage larva and Mycobacterium phage fionn) in the genus Tm4likevirus (new), family Siphoviridae, order Caudovirales 
2013.022bB create a new genus in the family Siphoviridae

2013.022cB name the new genus Tm4likevirus

2013.022dB designate Mycobacterium phage TM4 as the type species of the new genus

\subsection{3a-dB.A.v3.C5likevirus [42]}

2013.023aB create 2 species, Lactobacillus phage c5 and Lactobacillus phage LLKu, in the genus C5likevirus (new), family Siphoviridae, order Caudovirales

2013.023bB create a new genus in the family Siphoviridae

$2013.023 \mathrm{cB}$ name the new genus C5likevirus

$2013.023 \mathrm{~dB}$ designate Lactobacillus phage $c 5$ as the type species of the new genus

2013.024a-dB.A.v3.P23likevirus [43]

2013.024aB create 2 species, Thermus phage P2345 and Thermus phage P7426, in the genus P23likevirus (new), family Siphoviridae, order Caudovirales

2013.024bB create a new genus in the family Siphoviridae

$2013.024 \mathrm{cB}$ name the new genus P23likevirus

2013.024dB designate Thermus phage P2345 as the type species of the new genus

2013.025a-dB.A.v3.Phicbklikevirus [44]

$2013.025 \mathrm{aB}$ create 5 species (Caulobacter phage phicbk, Caulobacter phage magneto, Caulobacter phage swift, Caulobacter phage karma and Caulobacter phage rogue) in the genus Phicbklikevirus (new), family Siphoviridae, order Caudovirales

2013.025bB create a new genus in the family Siphoviridae

$2013.025 \mathrm{cB}$ name the new genus Phicbklikevirus

$2013.025 \mathrm{~dB}$ designate Caulobacter phage phicbk as the type species of the new genus

2013.026a-dB.A.v3.Sap6likevirus [45]

2013.026aB create 5 species (Enterococcus phage SAP6, Enterococcus phage BC611, Enterococcus phage IMEEF1, Streptococcus phage SPQS1 and Enterococcus phage VD13) in the genus Sap6likevirus (new), family Siphoviridae, order Caudovirales

2013.026bB create a new genus in the family Siphoviridae

2013.026cB name the new genus Sap6likevirus

2013.026dB designate Enterococcus phage SAP6 as the type species of the new genus

2013.027a-dB.A.v3.Tp2unalikevirus [46]

2013.027aB create 2 species, Bacillus phage TP21 and Bacillus phage BMBtp2, in the genus Tp2unalikevirus (new), family Siphoviridae, order Caudovirales

2013.027bB create a new genus in the family Siphoviridae

$2013.027 \mathrm{cB}$ name the new genus Tp2unalikevirus

$2013.027 \mathrm{~dB}$ designate Bacillus phage TP21 as the type species of the new genus

2013.028a-dB.A.v3.Xp10likevirus [47]

2013.028aB create 5 species (Xanthomonas phage Xp10, Xanthomonas phage OP1, Xanthomonas phage Xop411, Xanthomonas phage phil7 and Xanthomonas phage CP1) in the genus Xp10likevirus (new), family Siphoviridae, order Caudovirales

2013.028bB create a new genus in the family Siphoviridae

$2013.028 \mathrm{cB}$ name the new genus Xp10likevirus

2013.028dB designate Xanthomonas phage Xp10 as the type species of the new genus

2013.029a-dB.A.v3.Hk578likevirus [48]

2013.029aB create 5 species (Escherichia phage HK578, Sodalis phage SO-1, Shigella phage EP23, Enterobacteria phage JL1 and Enterobacteria phage SSL-2009a) in the genus Hk578likevirus (new), family Siphoviridae, order Caudovirales

2013.029bB create a new genus in the family Siphoviridae

$2013.029 \mathrm{cB}$ name the new genus Hk578likevirus

$2013.029 \mathrm{~dB}$ designate Escherichia phage HK578 as the type species of the new genus

2013.030a-dB.A.v3.Jerseylikevirus [49]

2013.030aB create 6 species (Salmonella phage jersey, Salmonella phage SETP3, Salmonella phage Ent1, Salmonella phage SE2, Salmonella phage wksl3 and Salmonella phage SS3e) in the genus Jerseylikevirus (new), family Siphoviridae, order Caudovirales

2013.030bB create a new genus in the family Siphoviridae

2013.030cB name the new genus Jerseylikevirus

$2013.030 \mathrm{~dB}$ designate Salmonella phage jersey as the type species of the new genus

2013.031a-dB.A.v3.Phie125likevirus [50] 
2013.031aB create 3 species (Burkholderia phage phie125, Burkholderia phage phi1026b and Burkholderia phage phi6442) in the genus Phie125likevirus (new), family Siphoviridae, order Caudovirales

$2013.031 \mathrm{bB}$ create a new genus in the family Siphoviridae

2013.031cB name the new genus Phie125likevirus

$2013.031 \mathrm{~dB}$ designate Burkholderia phage phie125 as the type species of the new genus

2013.032a-dB.A.v3.Wbetalikevirus [51]

2013.032aB create species Bacillus phage Wbeta in the genus Wbetalikevirus (new), family Siphoviridae, order Caudovirales

2013.032bB create a new genus in the family Siphoviridae

2013.032cB name the new genus Whetalikevirus

2013.032dB designate Bacillus phage Wbeta as the type species of the new genus

\subsection{3aB.A.v3.PhiC3unalikeviruses_2sp [52]}

2013.033aB create 2 species, Streptomyces phage phibt1 and Streptomyces phage TG1, in the genus PhiC3unalikevirus (new), family Siphoviridae, order Caudovirales

2013.034a-dB.A.v4.Sfilunalikevirus [53]

2013.034aB create 5 species (Streptococcus phage Sfi11, Streptococcus phage O1205, Streptococcus phage 2972, Streptococcus phage Alq132 and Streptococcus phage 858) in the genus Sfilunalikevirus (new), family Siphoviridae, order Caudovirales

2013.034bB create a new genus in the family Siphoviridae

$2013.034 \mathrm{cB}$ name the new genus Sfilunalikevirus

2013.034dB designate Streptococcus phage Sfill as the type species of the new genus

2013.035a-dB.A.v3.Skunalikevirus [54]

$2013.035 \mathrm{aB} \quad$ create 17 species (Lactococcus phage SK1, Lactococcus phage bIl170, Lactococcus phage jj50, Lactococcus phage 712, Lactococcus phage P008, Lactococcus phage Sl4, Lactococcus phage CB13, Lactococcus phage CB14, Lactococcus phage CB19, Lactococcus phage CB20, Lactococcus phage Bibb29, Lactococcus phage P2, Lactococcus phage Ascc273, Lactococcus phage Ascc191, Lactococcus phage Ascc281, Lactococcus phage Ascc532 and Lactococcus phage Ascc465) in the genus Skunalikevirus (new), family Siphoviridae, order Caudovirales

2013.035bB create a new genus in the family Siphoviridae

$2013.035 \mathrm{cB}$ name the new genus Skunalikevirus

2013.035dB designate Lactococcus phage SK1 as the type species of the new genus

2013.036a-dB.A.v3.Sfi21dtunalikevirus [55]

2013.036aB create 5 species (Streptococcus phage DT1, Streptococcus phage Sfi19, Streptococcus phage Sfi21, Streptococcus phage Abc2 and Streptococcus phage 7201) in the genus Sfi21dtunalikevirus (new), family Siphoviridae, order Caudovirales

2013.036bB create a new genus in the family Siphoviridae

$2013.036 \mathrm{cB}$ name the new genus Sfi21dtunalikevirus

2013.036dB designate Streptococcus phage DT1 as the type species of the new genus

2013.037a-dB.A.v3.Andromedalikevirus [56]

2013.037aB create 8 species (Bacillus phage andromeda, Bacillus phage eoghan, Bacillus phage taylor, Bacillus phage curly, Bacillus phage finn, Bacillus phage glittering, Bacillus phage blastoid and Bacillus phage riggi) in the genus Andromedalikevirus (new), family Siphoviridae, order Caudovirales

2013.037bB create a new genus in the family Siphoviridae

2013.037cB name the new genus Andromedalikevirus

$2013.037 \mathrm{~dB}$ designate Bacillus phage andromeda as the type species of the newus

2013.038a-dB.A.v4.Bcep22likevirus [57]

2013.038aB create 4 species (Burkholderia phage Bcep22, Burkholderia phage Bcepil02, Burkholderia phage Dc1 and Burkholderia phage Bcepmigl) in the genus Bcep22likevirus (new), family Podoviridae, order Caudovirales

2013.038bB create a new genus in the family Podoviridae

$2013.038 \mathrm{cB}$ name the new genus Bcep22likevirus

$2013.038 \mathrm{~dB}$ designate Burkholderia phage Bcep22 as the type species of the new genus

2013.039a-dB.A.v3.Chilikevirus [58]

2013.039aB create 5 species (Salmonella phage Chi, Salmonella phage SPN19, Salmonella phage iEPS5, Salmonella phage FSLSP088 and Salmonella phage FSLSP030) in the genus Chilikevirus (new), family Siphoviridae, order Caudovirales

2013.039bB create a new genus in the family Siphoviridae

$2013.039 \mathrm{cB}$ name the new genus Chilikevirus

2013.039dB designate Salmonella phage Chi as the type species of the new genus 


\subsection{0a-dB.A.v4.F116likevirus [59]}

2013.040aB create 2 species, Pseudomonas phage F116 and Pseudomonas phage H66, in the genus F116likevirus (new), family Podoviridae, order Caudovirales

2013.040bB create a new genus in the family Podoviridae

$2013.040 \mathrm{cB}$ name the new genus F116likevirus

2013.040dB designate Pseudomonas phage F116 as the type species of the new genus

2013.042a-lB.A.v3.Siphoviridae_3gen $[60,61]$

2013.042aB create 6 species (Staphylococcus phage 3a, Staphylococcus phage 42e, Staphylococcus phage 47, Staphylococcus phage Ipla35, Staphylococcus phage Slt and Staphylococcus phage Phi12) in the genus 3alikevirus (new), family Siphoviridae, order Caudovirales

2013.042bB create 3 species (Staphylococcus phage 77, Staphylococcus phage 13 and Staphylococcus phage Pvl108) in the genus 77likevirus (new), family Siphoviridae, order Caudovirales

2013.042cB create 31 species (Staphylococcus phage 11, Staphylococcus phage 187, Staphylococcus phage 29, Staphylococcus phage 37, Staphylococcus phage 52a, Staphylococcus phage 53, Staphylococcus phage 55, Staphylococcus phage 69, Staphylococcus phage 71, Staphylococcus phage 80, Staphylococcus phage 80alpha, Staphylococcus phage 85, Staphylococcus phage 88, Staphylococcus phage 92, Staphylococcus phage 96, Staphylococcus phage Cnph82, Staphylococcus phage Ew, Staphylococcus phage Ph15, Staphylococcus phage Phieta, Staphylococcus phage Phieta2, Staphylococcus phage Phieta3, Staphylococcus phage phimr11, Staphylococcus phage phimr25, Staphylococcus phage Ipla88, Staphylococcus phage Sap26, Staphylococcus phage X2, Staphylococcus phage phinm1, Staphylococcus phage phinm4, Staphylococcus phage Ipla5, Staphylococcus phage Ipla7 and Staphylococcus phage phinm2) in the genus Phietalikevirus (new), family Siphoviridae, order Caudovirales

$2013.042 \mathrm{~dB}$ create a new genus in the family Siphoviridae

$2013.042 \mathrm{eB}$ name the new genus 3alikevirus

2013.042fB designate Staphylococcus phage $3 a$ as the type species of the new genus

$2013.042 \mathrm{gB}$ create a new genus in the family Siphoviridae

2013.042hB name the new genus 77alikevirus

2013.042iB designate Staphylococcus phage 77 as the type species of the new genus

2013.042jB create a new genus in the family Siphoviridae

2013.042kB name the new genus Phietalikevirus

2013.0421B designate Staphylococcus phage Phieta as the type species of the new gen

\section{Vertebrate virus subcommittee}

2013.004aV.A.v2.Phlebovirus_sp [62]

2013.004aV create species SFTS virus in the genus Phlebovirus, family Bunyaviridae

2014.001aV.A.v1. Orthohepadnavirus_sp [63]

2014.001aV create species Bat hepatitis virus in genus Orthohepadnavirus, family Hepadnaviridae

\subsection{2aV.A.v2.Rotavirus_3sp [64]}

2014.002aV create 3 species (Rotavirus F, Rotavirus G, and Rotavirus H), in the genus Rotavirus, subfamily Sedoreovirinae, family Reoviridae

2014.004aV.A.v2.Tospovirus_sp [65]

$2014.004 \mathrm{aV}$ create species Iris yellow spot virus in the genus Tospovirus, family Bunyaviridae

2014.005aV.A.v2.Tospovirus_sp [66]

2014.005aV create species Watermelon bud necrosis virus in the genus Tospovirus, family Bunyaviridae

\subsection{7aV.A.v2.Nyavirus_sp [67]}

2014.007aV create species Sierra Nevada nyavirus in the genus Nyavirus, family Nyamiviridae, order Mononegavirales

\subsection{8a-hV.A.v6.Hepeviridae [68]}

2014.008aV create species Piscihepevirus A in the genus Piscihepevirus (new), family Hepeviridae

2014.008bV create 2 species (Orthohepevirus $C$ and Orthohepevirus D) in the genus Hepevirus (proposed name, Orthohepevirus) family Hepeviridae

$2014.008 \mathrm{cV}$ create a new genus in the family Hepeviridae

2014.008dV name the genus Piscihepevirus

$2014.008 \mathrm{eV}$ designate Piscihepevirus A as type species of the new genus

$2014.008 \mathrm{fV}$ remove species Avian hepatitis E virus from its unassigned position within the family Hepeviridae

$2014.008 \mathrm{gV}$ reassign the above species to the genus Hepevirus (proposed name, Orthohepevirus)

$2014.008 \mathrm{hV}$ (i) Change the name of the genus Hepevirus to Orthohepevirus. (ii) Change the name of the species Hepatitis E virus to Orthohepevirus A. (iii) Change the name of the species Avian hepatitis E virus to Orthohepevirus B. 
2014.010a,bV.A.v2.Bornaviridae_spren [69, 70]

2014.010aV create 4 species (Psittaciform 1 bornavirus, Passeriform 1 bornavirus, Passeriform 2 bornavirus and Waterbird 1 bornavirus) in the genus Bornavirus, family Bornaviridae

2014.010bV In the genus Bornavirus, change the name of species Borna disease virus to Mammalian 1 bornavirus

2014.011a-dV.A.v2.Reptarenavirus [71]

2014.011aV create 3 species (Alethinophid 1 reptarenavirus, Alethinophid 2 reptarenavirus and Alethinophid 3 reptarenavirus) in the genus Reptarenavirus (new), family Arenaviridae

2014.011bV create a new genus in the family Arenaviridae

$2014.011 \mathrm{cV}$ name the genus Reptarenavirus

$2014.011 \mathrm{dV}$ designate Alethinophid 1 reptarenavirus as type species of the new genus

2014.012aV.A.v3.Arenavirus_ren [72]

2014.012aV in the family Arenaviridae, change the name of genus Arenavirus to Mammarenavirus and convert the names of its constituent species to a binomial format, by changing the word virus to mammarenavirus in each existing name, [example: Allpahuayo virus (current name) changed to Allpahuayo mammarenavirus (new name)]

2014.013aV.A.v3. Mammarenavirus_2sp [73]

2014.013aV create species Lunk mammarenavirus and Merino Walk mammarenavirus in the genus Arenavirus (proposed name Mammarenavirus), family Arenaviridae

2014.014aV.A.v2.Cardiovirus_sp [74]

2014.014aV create species Cardiovirus $C$ within the genus Cardiovirus, family Picornaviridae

2014.015a-dV.A.v3.Kunsagivirus [75]

2014.015aV create species Kunsagivirus A in genus Kunsagivirus (new), family Picornaviridae

2014.015bV create a new genus in the family Picornaviridae

$2014.015 \mathrm{cV}$ name the genus Kunsagivirus

2014.015dV designate Kunsagivirus $A$ as type species of the new genus

2014.016aV.A.v1.Picornaviridae_spren [76]

2014.016aV rename 12 species in the family Picornaviridae as follows:

\section{Genus}

Avihepatovirus

Cardiovirus

Cardiovirus

Erbovirus

Hepatovirus

Parechovirus

Parechovirus

Sapelovirus

Sapelovirus

Senecavirus

Teschovirus

Tremovirus

\section{Old name}

Duck hepatitis A virus

Encephalomyocarditis virus

Theilovirus

Equine rhinitis $B$ virus

Hepatitis A virus

Human parechovirus

Ljungan virus

Porcine sapelovirus

Simian sapelovirus

Seneca Valley virus

Porcine teschovirus

Avian encephalomyelitis virus

New name
Avihepatovirus A
Cardiovirus A
Cardiovirus B
Erbovirus A
Hepatovirus A
Parechovirus A
Parechovirus B
Sapelovirus A
Sapelovirus B
Senecavirus A
Teschovirus A
Tremovirus A

New name

Avihepatovirus A

Cardiovirus A

Cardiovirus $B$

Erbovirus $A$

Hepatovirus A

Parechovirus A

Parechovirus $B$

Sapelovirus A

Sapelovirus $B$

Teschovirus A

\subsection{7a-dV.A.v3.Sakobuvirus [77]}

2014.017aV create species Sakobuvirus A in genus Sakobuvirus (new), family Picornaviridae

2014.017bV create a new genus in the family Picornaviridae

$2014.017 \mathrm{cV}$ name the genus Sakobuvirus

$2014.017 \mathrm{dV}$ designate Sakobuvirus A as type species of the new genus

\subsection{8a-dV.A.v3.Sicinivirus [78]}

$2014.018 \mathrm{aV}$

create species Sicinivirus A in genus Sicinivirus (new), family Picornaviridae

$2014.018 b \mathrm{~b}$

$2014.018 \mathrm{cV}$

create a new genus in the family Picornaviridae

$2014.018 \mathrm{dV}$

name the genus Sicinivirus

designate Sicinivirus $A$ as type species of the new genus 
All of the above taxonomic proposals were approved by the members who voted, mostly unanimously and always $>96 \%$. The return rate of votes was approximately $69 \%$. These taxonomic changes are now part of the official ICTV taxonomy and represent changes to the taxonomy published in the Ninth Report [79] and are in addition to changes approved in February 2012 [80], February 2013 [81] and March 2014 [82]. An up-to-date list of all approved taxa can be found on the ICTV online web site: http://www.ictvonline.org/msl.htm.

The authors declare no conflicts of interest. None of work reported involved research on human participants or animals. All authors have contributed to this work and agreed to its publication.

\section{References}

1. Arif B, Herniou E, Thézé J, Nakai M, Takatsuka J (2014) ICTV taxonomic proposal 2013.002aI.A.v3.Betaentomopoxvirus_3sp. Create 3 new species in the genus Betaentomopoxvirus, family Poxviridae. http://www.ictvonline.org/proposals-14/2013.002aI. A.v3.Betaentomopoxvirus_3sp.pdf

2. Adams MJ (2014) ICTV taxonomic proposal 2014. 001aI.A.v1.Entomopoxvirus-spcorr. Correct species names in the subfamily Entomopoxvirinae, family Poxviridae. http://www.ict vonline.org/proposals-14/2014.001aI.A.v1.Entomopoxvirus-spcorr. pdf

3. Harrison RL, Herniou EA, Theilmann DA, Becnel JJ, Arif B, Jehle JA, Burand JP, van Oers M (2014) ICTV taxonomic proposal 2014.002aI.A.v1.Alphabaculovirus-sprem. Remove 1 species from the genus Alphabaculovirus, family Baculoviridae. http://www.ictvonline.org/proposals-14/2014.002aI.A.v1.Alpha baculovirus-sprem.pdf

4. Rochon D, Scheets K, White A, Martelli G, Rubino L (2014) ICTV taxonomic proposal 2013.010a, bP.A.v2.Umbravirus_move. Move genus Umbravirus into family Tombusviridae. http://www.ictvonline.org/proposals-14/2013.010a,bP.A.v2.Umbra virus_move.pdf

5. Adams MJ, Adkins S, Bragard C, Gilmer D, Li D, MacFarlane SA, Man WS, Melcher U, Ratti C, Ryu KH (2014) ICTV taxonomic proposal 2014.001aP.A.v1.Tobamovirus_2sp. Create 2 new species in the genus Tobamovirus, family Virgaviridae. http://www.ictvonline.org/proposals-14/2014.001aP.A.v1.Toba movirus_2sp.pdf

6. Verhoeven JThJ, Owens RA (2014) ICTV taxonomic proposal 2014.002aP.A.v1.Pospiviroid_sprem. Reclassification of Mexican papita viroid as a strain of Tomato planta macho viroid. http:// www.ictvonline.org/proposals-14/2014.002aP.A.v1.Pospiviroid_ sprem.pdf

7. Flores R, Verhoeven JThJ (2014) ICTV taxonomic proposal 2014.003aP.A.v2.Hostuviroid_sp. Create 1 new species in the genus Hostuviroid, family Pospiviroidae. http://www.ictvonline. org/proposals-14/2014.003aP.A.v2.Hostuviroid_sp.pdf

8. Verbeek M, Dullemans AM, van der Vlugt RAA (2014) ICTV taxonomic proposal 2014.004aP.A.v2.Torradovirus_sp. Create 1 new species in the genus Torradovirus, family Secoviridae, order Picornavirales. http://www.ictvonline.org/proposals-14/2014. 004aP.A.v2.Torradovirus_sp.pdf

9. Rabenstein F, Adams MJ, Kreuze JF, Lopez-Moya JJ, Ohshima K, Stenger DC, Wang A, Wylie S, Zerbini FM (2014) ICTV taxonomic proposal 2014.005aP.A.v1.Tritimovirus_sp. Create 1 new species in the genus Tritimovirus, family Potyviridae. http://www.ictvonline. org/proposals-14/2014.005aP.A.v1.Tritimovirus_sp.pdf

10. Scheets K, Hernandez C, Jordan R, White A (2014) ICTV taxonomic proposal 2014.006aP.A.v2.Tombusviridae_4sp. Create 4 new species unassigned in the family Tombusviridae. http:// www.ictvonline.org/proposals-14/2014.006aP.A.v2.Tombusvir idae_4sp.pdf

11. Scheets K (2014) ICTV taxonomic proposal 2014.007aP.A.v1.Panicovirus_sp. Create 1 new species in the genus Panicovirus, family Tombusviridae. http://www.ictvonline.org/proposals-14/ 2014.007aP.A.v1.Panicovirus_sp.pdf

12. Scheets K, Melcher U (2014) ICTV taxonomic proposal 2014.008aP.A.v3.Tombusviridae_sp. Create 1 new species unassigned in the family Tombusviridae. http://www.ictvonline. org/proposals-14/2014.008aP.A.v3.Tombusviridae_sp.pdf

13. Rabenstein F, Adams MJ, Kreuze JF, Lopez-Moya JJ, Ohshima K, Stenger DC, Wang A, Wylie S, Zerbini FM (2014) ICTV taxonomic proposal 2014.009aP.A.v4.Potyvirus_12sp. Create 12 new species in the genus Potyvirus, family Potyviridae. http://www. ictvonline.org/proposals-14/2014.009aP.A.v4.Potyvirus_12sp.pdf

14. Geering ADW (2014) ICTV taxonomic proposal 2014.010adP.A.v2.Rosadnavirus. Create new genus with 1 new species in the family Caulimoviridae. http://www.ictvonline.org/proposals14/2014.010a-dP.A.v2.Rosadnavirus.pdf

15. Geering ADW (2014) ICTV taxonomic proposal 2014.011aP. A.v2.Caulimovirus_sp. Create 1 new species in the genus Caulimovirus, family Caulimoviridae. http://www.ictvonline.org/pro posals-14/2014.011aP.A.v2.Caulimovirus_sp.pdf

16. Geering ADW (2014) ICTV taxonomic proposal 2014.012aP.A.v2.Badnavirus_7sp. Create 7 new species in the genus Badnavirus, family Caulimoviridae. http://www.ictvonline. org/proposals-14/2014.012aP.A.v2.Badnavirus_7sp.pdf

17. Elbeaino T (2014) ICTV taxonomic proposal 2014. 013aP.A.v2.Emaravirus_sp. Create 1 new species in the genus Emaravirus. http://www.ictvonline.org/proposals-14/2014.013aP. A.v2.Emaravirus_sp.pdf

18. Kropinski AM, Lavigne R, Adriaenssens E, Nash J (2014) ICTV taxonomic proposal 2012.002a-dB.A.v3.Corndoglikevirus. Create new genus with 2 new species in the family Siphoviridae, order Caudovirales. http://www.ictvonline.org/proposals-14/ 2012.002a-dB.A.v3.Corndoglikevirus.pdf

19. Kropinski AM, Lavigne R, Adriaenssens E (2014) ICTV taxonomic proposal 2012.003a-dB.A.v3.D3112likevirus. Create new genus with 8 new species in the family Siphoviridae, order Caudovirales. http://www.ictvonline.org/proposals-14/2012. 003a-dB.A.v3.D3112likevirus.pdf

20. Dyall-Smith M, Porter K, Tang S-L, Pawlowski A, Rissanen I, Bamford JKH, Krupovic M, Jalasvuori M (2014) ICTV taxonomic proposal 2013.001a-oB.A.v5.Sphaerolipoviridae. Create a new family Sphaerolipoviridae with 3 new genera and 6 new species. http://www.ictvonline.org/proposals-14/2013.001a-oB. A.v5.Sphaerolipoviridae.pdf

21. Pawlowski A, Rissanen I, Bamford JKH, Krupovic M, Jalasvuori M (2014) Gammasphaerolipovirus, a newly proposed bacteriophage genus, unifies viruses of halophilic archaea and thermophilic bacteria within the novel family Sphaerolipoviridae. Arch Virol 159:1541-1554

22. Kropinski AM, Lavigne R, Ackermann H-W, Szymanski C, Javed MA, Connerton I, Timms A, Azeredo J, Carvalho CM., Hertwig S, Hammerl JA (2014) ICTV taxonomic proposal 2013.004a-kB.A.v4.Eucampyvirinae. Create 2 new genera with 4 and 3 new species in a new subfamily in the family Myoviridae, order Caudovirales. http://www.ictvonline.org/proposals-14/ 2013.004a-kB.A.v4.Eucampyvirinae.pdf

23. Javed MA, Ackermann HW, Azeredo J, Carvalho CM, Connerton I, Evoy S, Hammerl JA, Hertwig S, Lavigne R, Singh A, 
Szymanski CM, Timms A, Kropinski AM (2014) A suggested classification for two groups of Campylobacter myoviruses. Arch Virol 159:181-190

24. Adriaenssens E, Kropinski AM, Lavigne R, Horsburgh M (2014) ICTV taxonomic proposal 2013.005a-dB.A.v3.Phiflikevirus. Create new genus with 3 new species in the family Siphoviridae, order Caudovirales. http://www.ictvonline.org/proposals-14/ 2013.005a-dB.A.v3.Phifllikevirus.pdf

25. Adriaenssens E, Kropinski AM, Lavigne R, Van Melderen L (2014) ICTV taxonomic proposal 2013.006a-dB.A.v3.Iebhlikevirus. Create new genus with 2 new species in the family Siphoviridae, order Caudovirales. http://www.ictvonline.org/pro posals-14/2013.006a-dB.A.v3.Iebhlikevirus.pdf

26. Adriaenssens E, Kropinski AM, Lavigne R (2014) ICTV taxonomic proposal 2013.007a-dB.A.v3.Halolikevirus. Create new genus with 2 new species in the family Siphoviridae, order Caudovirales. http://www.ictvonline.org/proposals-14/2013.007adB.A.v3.Halolikevirus.pdf

27. Adriaenssens E, Kropinski AM, Lavigne R (2014) ICTV taxonomic proposal 2013.008a-dB.A.v3.Che8likevirus. Create new genus with 28 new species in the family Siphoviridae, order Caudovirales. http://www.ictvonline.org/proposals-14/2013.008adB.A.v3.Che8likevirus.pdf

28. Adriaenssens E, Kropinski AM, Lavigne R (2014) ICTV taxonomic proposal 2013.009a-dB.A.v3.Bignuzlikevirus. Create new genus with 2 new species in the family Siphoviridae, order Caudovirales. http://www.ictvonline.org/proposals-14/2013.009adB.A.v3.Bignuzlikevirus.pdf

29. Adriaenssens E, Kropinski AM, Lavigne R, Coffey A (2014) ICTV taxonomic proposal 2013.010a-dB.A.v3.Phijlunalikevirus. Create new genus with 3 new species in the family Siphoviridae, order Caudovirales. http://www.ictvonline.org/proposals-14/2013. 010a-dB.A.v3.Phijlunalikevirus.pdf

30. Adriaenssens E, Kropinski AM, Lavigne R, Nash J (2014) ICTV taxonomic proposal 2013.011a-dB.A.v3.Barnyardlikevirus. Create new genus with 4 new species in the family Siphoviridae, order Caudovirales. http://www.ictvonline.org/proposals-14/ 2013.011a-dB.A.v3.Barnyardlikevirus.pdf

31. Adriaenssens E, Kropinski AM, Lavigne R, Nash J (2014) ICTV taxonomic proposal 2013.012a-dB.A.v3.Charlielikevirus. Create new genus with 2 new species in the family Siphoviridae, order Caudovirales. http://www.ictvonline.org/proposals-14/2013.012adB.A.v3.Charlielikevirus.pdf

32. Adriaenssens E, Kropinski AM, Lavigne R, Nash J (2014) ICTV taxonomic proposal 2013.013a-dB.A.v3.Che9clikevirus. Create new genus with 3 new species in the family Siphoviridae, order Caudovirales. http://www.ictvonline.org/proposals-14/2013.013adB.A.v3.Che9clikevirus.pdf

33. Adriaenssens E, Kropinski AM, Lavigne R, Nash J (2014) ICTV taxonomic proposal 2013.014a-dB.A.v3.Cjwunalikevirus. Create new genus with 9 new species in the family Siphoviridae, order Caudovirales. http://www.ictvonline.org/proposals-14/2013.014adB.A.v3.Cjwunalikevirus.pdf

34. Adriaenssens E, Kropinski AM, Lavigne R, Edwards R (2014) ICTV taxonomic proposal 2013.015a-dB.A.v3.D3likevirus. Create new genus with 2 new species in the family Siphoviridae, order Caudovirales. http://www.ictvonline.org/proposals-14/ 2013.015a-dB.A.v3.D3likevirus.pdf

35. Adriaenssens E, Kropinski AM, Lavigne R, Nash J (2014) ICTV taxonomic proposal 2013.016aB.A.v3.L5likevirus_62sp. Create 62 new species in the genus L5likevirus, family Siphoviridae, order Caudovirales. http://www.ictvonline.org/proposals-14/ 2013.016aB.A.v3.L5likevirus_62sp.pdf

36. Adriaenssens E, Kropinski AM, Lavigne R, Nash J (2014) ICTV taxonomic proposal 2013.017a-dB.A.v3.Bronlikevirus. Create new genus with 4 new species in the family Siphoviridae, order
Caudovirales. http://www.ictvonline.org/proposals-14/2013. 017a-dB.A.v3.Bronlikevirus.pdf

37. Adriaenssens E, Kropinski AM, Lavigne R, Nash J (2014) ICTV taxonomic proposal 2013.018a-dB.A.v3.Omegalikevirus. Create new genus with 6 new species in the family Siphoviridae, order Caudovirales. http://www.ictvonline.org/proposals-14/2013.018adB.A.v3.Omegalikevirus.pdf

38. Adriaenssens E, Kropinski AM, Lavigne R, Nash J (2014) ICTV taxonomic proposal 2013.019a-dB.A.v4.Pbiunalikevirus. Create new genus with 1 new species in the family Siphoviridae, order Caudovirales. http://www.ictvonline.org/proposals-14/2013.019adB.A.v4.Pbiunalikevirus.pdf

39. Adriaenssens E, Kropinski AM, Lavigne R, Nash J (2014) ICTV taxonomic proposal 2013.020a-dB.A.v4.Pgonelikevirus. Create new genus with 12 new species in the family Siphoviridae, order Caudovirales. http://www.ictvonline.org/proposals-14/2013.020adB.A.v4.Pgonelikevirus.pdf

40. Adriaenssens E, Kropinski AM, Lavigne R, Nash J (2014) ICTV taxonomic proposal 2013.021a-dB.A.v3.Reylikevirus. Create new genus with 2 new species in the family Siphoviridae, order Caudovirales. http://www.ictvonline.org/proposals-14/2013.021adB.A.v3.Reylikevirus.pdf

41. Adriaenssens E, Kropinski AM, Lavigne R, Nash J (2014) ICTV taxonomic proposal 2013.022a-dB.A.v3.Tm4likevirus. Create new genus with 9 new species in the family Siphoviridae, order Caudovirales. http://www.ictvonline.org/proposals-14/2013.022adB.A.v3.Tm4likevirus.pdf

42. Adriaenssens E, Kropinski AM, Lavigne R, Nash J (2014) ICTV taxonomic proposal 2013.023a-dB.A.v3.C5likevirus. Create new genus with 2 new species in the family Siphoviridae, order Caudovirales. http://www.ictvonline.org/proposals-14/2013.023adB.A.v3.C5likevirus.pdf

43. Adriaenssens E, Kropinski AM, Lavigne R, Edwards R (2014) ICTV taxonomic proposal 2013.024a-dB.A.v3.P23likevirus. Create new genus with 2 new species in the family Siphoviridae, order Caudovirales. http://www.ictvonline.org/proposals-14/2013. 024a-dB.A.v3.P23likevirus.pdf

44. Adriaenssens E, Kropinski AM, Lavigne R (2014) ICTV taxonomic proposal 2013.025a-dB.A.v3.Phicbklikevirus. Create new genus with 5 new species in the family Siphoviridae, order Caudovirales. http://www.ictvonline.org/proposals-14/2013.025adB.A.v3.Phicbklikevirus.pdf

45. Adriaenssens E, Kropinski AM, Lavigne R (2014) ICTV taxonomic proposal 2013.026a-dB.A.v3.Sap6likevirus. Create new genus with 5 new species in the family Siphoviridae, order Caudovirales. http://www.ictvonline.org/proposals-14/2013.026adB.A.v3.Sap6likevirus.pdf

46. Adriaenssens E, Kropinski AM, Lavigne R, Klumpp J (2014) ICTV taxonomic proposal 2013.027a-dB.A.v3.Tp2unalikevirus. Create new genus with 2 new species in the family Siphoviridae, order Caudovirales. http://www.ictvonline.org/proposals-14/ 2013.027a-dB.A.v3.Tp2unalikevirus.pdf

47. Adriaenssens E, Kropinski AM, Lavigne R, Edwards R (2014) ICTV taxonomic proposal 2013.028a-dB.A.v3.Xp10likevirus. Create new genus with 5 new species in the family Siphoviridae, order Caudovirales. http://www.ictvonline.org/proposals-14/ 2013.028a-dB.A.v3.Xp10likevirus.pdf

48. Refardt D, Ackermann H-W, Adriaenssens E, Kropinski AM, Lavigne R (2014) ICTV taxonomic proposal 2013.029adB.A.v3.Hk578likevirus. Create new genus with 5 new species in the family Siphoviridae, order Caudovirales. http://www.ictvon line.org/proposals-14/2013.029a-dB.A.v3.Hk578likevirus.pdf

49. Ackermann H-W, Adriaenssens E, Moineau S, Kropinski AM, Lavigne R, De Lappe N (2014) ICTV taxonomic proposal 2013.030a-dB.A.v3.Jerseylikevirus. Create new genus with 6 new species in the family Siphoviridae, order Caudovirales. 
http://www.ictvonline.org/proposals-14/2013.030a-dB.A.v3.Jer seylikevirus.pdf

50. Adriaenssens E, Kropinski AM, Lavigne R, Edwards R (2014) ICTV taxonomic proposal 2013.031a-dB.A.v3.Phie125likevirus. Create new genus with 3 new species in the family Siphoviridae, order Caudovirales. http://www.ictvonline.org/proposals-14/ 2013.031a-dB.A.v3.Phie125likevirus.pdf

51. Adriaenssens E, Kropinski AM, Lavigne R, Edwards R (2014) ICTV taxonomic proposal 2013.032a-dB.A.v3.Wbetalikevirus. Create new genus with 1 new species in the family Siphoviridae, order Caudovirales. http://www.ictvonline.org/proposals-14/ 2013.032a-dB.A.v3.Wbetalikevirus.pdf

52. Adriaenssens E, Kropinski AM, Lavigne R (2014) ICTV taxonomic proposal 2013.033aB.A.v3.PhiC3unalikeviruses_2sp. Create 2 new species in the genus PhiC3unalikevirus, family Siphoviridae, order Caudovirales. http://www.ictvonline.org/pro posals-14/2013.033aB.A.v3.PhiC3unalikeviruses_2sp.pdf

53. Adriaenssens E, Kropinski AM, Lavigne R, Nash J (2014) ICTV taxonomic proposal 2013.034a-dB.A.v4.Sfilunalikevirus. Create new genus with 5 new species in the family Siphoviridae, order Caudovirales. http://www.ictvonline.org/proposals-14/2013. 034a-dB.A.v4.Sfilunalikevirus.pdf

54. Adriaenssens E, Kropinski AM, Lavigne R, Edwards R (2014) ICTV taxonomic proposal 2013.035a-dB.A.v3.Skunalikevirus. Create new genus with 17 new species in the family Siphoviridae, order Caudovirales. http://www.ictvonline.org/proposals-14/ 2013.035a-dB.A.v3.Skunalikevirus.pdf

55. Adriaenssens E, Kropinski AM, Lavigne R, Nash J, Moineau S, Brüssow H (2014) ICTV taxonomic proposal 2013.036adB.A.v3.Sfi21dtunalikevirus. Create new genus with 5 new species in the family Siphoviridae, order Caudovirales. http://www. ictvonline.org/proposals-14/2013.036a-dB.A.v3.Sfi21dtunali kevirus.pdf

56. Temple LM, Kropinski AM, Lavigne R, Adriaenssens E (2014) ICTV taxonomic proposal 2013.037a-dB.A.v3.Andromedalikevirus. Create new genus with 8 new species in the family Siphoviridae, order Caudovirales. http://www.ictvonline.org/pro posals-14/2013.037a-dB.A.v3.Andromedalikevirus.pdf

57. Kropinski AM, Adriaenssens E, Lavigne R, Gill J, Dennis J (2014) ICTV taxonomic proposal 2013.038a-dB.A.v4.Bcep22likevirus. Create new genus with 4 new species in the family Podoviridae, order Caudovirales. http://www.ictvonline.org/pro posals-14/2013.038a-dB.A.v4.Bcep22likevirus.pdf

58. Ryu S, Kropinski AM, Lavigne R, Hendrix R, Casjens S, Adriaenssens E (2014) ICTV taxonomic proposal 2013.039adB.A.v3.Chilikevirus. Create new genus with 5 new species in the family Siphoviridae, order Caudovirales. http://www.ictvon line.org/proposals-14/2013.039a-dB.A.v3.Chilikevirus.pdf

59. Ackermann H-W, Kropinski AM, Adriaenssens E, Lavigne R (2014) ICTV taxonomic proposal 2013.040a-dB.A.v4.F116likevirus. Create new genus with 2 new species in the family Podoviridae, order Caudovirales. http://www.ictvonline.org/pro posals-14/2013.040a-dB.A.v4.F116likevirus.pdf

60. Adriaenssens E, Guttiérez D, García P, Kropinski AM, Lavigne R (2014) ICTV taxonomic proposal 2013.042a-1B.A.v3.Siphoviridae_3gen. Create 3 new genera with 6, 3 and 31 new species in the family Siphoviridae, order Caudovirales. http://www.ictvon line.org/proposals-14/2013.042a-1B.A.v3.Siphoviridae_3gen.pdf

61. Gutiérrez D, Adriaenssens EM, Martínez B, Rodríguez A, Lavigne R, Kropinski AM, García P (2014) Three proposed new bacteriophage genera of staphylococcal phages: "3alikevirus", "77likevirus" and "Phietalikevirus". Arch Virol 159:389-398

62. Li D, Yu X, Liang M (2014) ICTV taxonomic proposal 2013.004aV.A.v2.Phlebovirus_sp. Create 1 new species in the genus Phlebovirus, family Bunyaviridae. http://www.ictvonline. org/proposals-14/2013.004aV.A.v2.Phlebovirus_sp.pdf
63. Tu C, He B (2014) ICTV taxonomic proposal 2014.001aV.A.v1.Orthohepadnavirus_sp. Create 1 new species in the genus Orthohepadnavirus, family Hepadnaviridae. http:// www.ictvonline.org/proposals-14/2014.001aV.A.v1.Orthohepadna virus_sp.pdf

64. Matthijnssens J, Johne R, Desselberger U (2014) ICTV taxonomic proposal 2014.002aV.A.v2.Rotavirus_3sp. Create 3 new species in the genus Rotavirus, family Reoviridae. http://www. ictvonline.org/proposals-14/2014.002aV.A.v2.Rotavirus_3sp.pdf

65. Pappu HR, Bag S (2014) ICTV taxonomic proposal 2014.004aV.A.v2.Tospovirus_sp. Create 1 new species in the genus Tospovirus, family Bunyaviridae. http://www.ictvonline. org/proposals-14/2014.004aV.A.v2.Tospovirus_sp.pdf

66. Jain RK, Mandal B, Pappu HR, Holkar SK (2014) ICTV taxonomic proposal 2014.005aV.A.v2.Tospovirus_sp. Create 1 new species in the genus Tospovirus, family Bunyaviridae. http:// www.ictvonline.org/proposals-14/2014.005aV.A.v2.Tospovirus_ sp.pdf

67. Tesh RB, Ghedin E, Vasilakis N, Rogers MB, Cui L, Fitch A, Popov V, Pravassos da Rosa APA (2014) ICTV taxonomic proposal 2014.007aV.A.v2.Nyavirus_sp. Create 1 new species in the genus Nyavirus, family Nyamiviridae, order Mononegavirales. http://www. ictvonline.org/proposals-14/2014.007aV.A.v2.Nyavirus_sp.pdf

68. Purdy MA, Smith DB, Simmonds P, Emerson SU, Harrison T, Meng X-J, Okamoto H, van der Poel, WHM, Jameel S (2014) ICTV taxonomic proposal 2014.008a-hV.A.v6.Hepeviridae. Reorganization of the family Hepeviridae. http://www.ictvonline. org/proposals-14/2014.008a-hV.A.v6.Hepeviridae.pdf

69. Kuhn JH, Dürrwald R, Bao Y, Briese T, Carbone K, Clawson AN, Garten W, Jahrling PB, Kolodziejek J, Rubbenstroth D, Schwemmle M, Tomonaga K, Weissenböck H, Nowotny N (2014) ICTV taxonomic proposal 2014.010a, bV.A.v2.Bornaviridae_spren. Create 4 new species and rename an existing species in the genus Bornavirus, family Bornaviridae. http:// www.ictvonline.org/proposals-14/2014.010a,bV.A.v2.Bornavir idae_spren.pdf

70. Kuhn JH, Dürrwald R, Bào Y, Briese T, Carbone K, Clawson AN, deRisi JL, Garten W, Jahrling PB, Kolodziejek J, Rubbenstroth D, Schwemmle M, Stenglein M, Tomonaga K, Weissenböck H, Nowotny N (2015) Taxonomic reorganization of the family Bornaviridae. Arch Virol 160:621-632

71. Stenglein MD, DeRisi JL, Bao Y, Hepojoki J, Sironen T, Vapalahti O, Hetzel U (2014) ICTV taxonomic proposal 2014.011adV.A.v2.Reptarenavirus. Create new genus with 3 new species in the family Arenaviridae. http://www.ictvonline.org/proposals-14/ 2014.011a-dV.A.v2.Reptarenavirus.pdf

72. Buchmeier MJ, Charrel R, Clegg CS, de la Torre JC, Emonet S, Gonzalez J-P, Kuhn JH, Lukashevich IS, Peters CJ, Radoshitzky SR, Romanowski V, Salvato MS (2014) ICTV taxonomic proposal 2014.012aV.A.v3.Arenavirus_ren. Rename genus and all species in the family Arenaviridae. http://www.ictvonline.org/ proposals-14/2014.012aV.A.v3.Arenavirus_ren.pdf

73. Bao Y, Buchmeier MJ, Charrel R, Clegg CS, de la Torre JC, Emonet S, Gonzalez J-P, Kuhn JH, Lukashevich IS, Peters CJ, Radoshitzky SR, Romanowski V, Salvato MS (2014) ICTV taxonomic proposal 2014.013aV.A.v3.Mammarenavirus_2sp. Create 2 new species in the genus Arenavirus (proposed Mammarenavirus), family Arenaviridae. http://www.ictvonline.org/ proposals-14/2014.013aV.A.v3.Mammarenavirus_2sp.pdf

74. Knowles NJ, Delwart E, Gorbalenya AE, Hovi T, Hyypiä T, King AMQ, Lindberg AM, Pallansch MA, Palmenberg AC, Reuter G, Simmonds P, Skern T, Stanway G, Yamashita T, Zell R (2014) ICTV taxonomic proposal 2014.014aV.A.v2.Cardiovirus_sp. Create 1 new species in the genus Cardiovirus, family Picornaviridae, order Picornavirales. http://www.ictvonline.org/pro posals-14/2014.014aV.A.v2.Cardiovirus_sp.pdf 
75. Knowles NJ, Delwart E, Gorbalenya AE, Hovi T, Hyypiä T, King AMQ, Lindberg AM, Pallansch MA, Palmenberg AC, Reuter G, Simmonds P, Skern T, Stanway G, Yamashita T, Zell R (2014) ICTV taxonomic proposal 2014.015a-dV.A.v3.Kunsagivirus. Create new genus with 1 new species in the family Picornaviridae, order Picornavirales. http://www.ictvonline.org/pro posals-14/2014.015a-dV.A.v3.Kunsagivirus.pdf

76. Knowles NJ, Delwart E, Gorbalenya AE, Hovi T, Hyypiä T, King AMQ, Lindberg AM, Pallansch MA, Palmenberg AC, Reuter G, Simmonds P, Skern T, Stanway G, Yamashita T, Zell R (2014) ICTV taxonomic proposal 2014.016aV.A.v1.Picornaviridae_spren. Rename 12 species in the family Picornaviridae, order Picornavirales. http://www.ictvonline.org/proposals-14/ 2014.016aV.A.v1.Picornaviridae_spren.pdf

77. Knowles NJ, Delwart E, Gorbalenya AE, Hovi T, Hyypiä T, King AMQ, Lindberg AM, Pallansch MA, Palmenberg AC, Reuter G, Simmonds P, Skern T, Stanway G, Yamashita T, Zell R (2014) ICTV taxonomic proposal 2014.017a-dV.A.v3.Sakobuvirus. Create new genus with 1 new species in the family Picornaviridae, order Picornavirales. http://www.ictvonline.org/pro posals-14/2014.017a-dV.A.v3.Sakobuvirus.pdf
78. Knowles NJ, Delwart E, Gorbalenya AE, Hovi T, Hyypiä T, King AMQ, Lindberg AM, Pallansch MA, Palmenberg AC, Reuter G, Simmonds P, Skern T, Stanway G, Yamashita T, Zell R (2014) ICTV taxonomic proposal 2014.018a-dV.A.v3.Sicinivirus. Create new genus with 1 new species in the family Picornaviridae, order Picornavirales. http://www.ictvonline.org/proposals-14/2014. 018a-dV.A.v3.Sicinivirus.pdf

79. King AMQ, Adams MJ, Carstens EB, Lefkowitz EJ (2011) Virus taxonomy. Ninth Report of the International Committee on Taxonomy of Viruses. Elsevier Academic Press, London, San Diego

80. Adams MJ, Carstens EB (2012) Ratification vote on taxonomic proposals to the International Committee on Taxonomy of Viruses (2012). Arch Virol 157:1411-1422

81. Adams MJ, King AMQ, Carstens EB (2013) Ratification vote on taxonomic proposals to the International Committee on Taxonomy of Viruses (2013). Arch Virol 158:2023-2030

82. Adams MJ, Lefkowitz EJ, King AMQ, Carstens EB (2014) Ratification vote on taxonomic proposals to the International Committee on Taxonomy of Viruses (2014). Arch Virol 159:2831-2841 